\title{
Generation of graded porous structures by control of process parameters in the selective laser melting of a fixed ratio salt-metal feedstock
}

\author{
Nesma T. Aboulkhair ${ }^{\text {a* }}$, G. Zhao ${ }^{\text {a }}$, R. J. M. Hague ${ }^{\text {a }}$, A.R. Kennedy ${ }^{\text {, }}$ I. A. Ashcroft ${ }^{\text {a }, ~ A . T . ~}$ \\ Clare $^{\mathrm{c}, \mathrm{d}}$ \\ ${ }^{a}$ Centre for Additive Manufacturing, Faculty of Engineering, University of Nottingham, \\ Nottingham, NG8 1BB, United Kingdom \\ ${ }^{\mathrm{b}}$ Engineering Department, Lancaster University, Lancaster LA1 4YW, United Kingdom \\ c Advanced Component Engineering Laboratory (ACEL), University of Nottingham, \\ Nottingham, NG7 2RD, United Kingdom \\ ${ }^{d}$ Department of Mechanical, Materials and Manufacturing Engineering, Faculty of Science \\ and Engineering, University of Nottingham China, 199 Taikang East Road, University Park, \\ Ningbo, 315100, China
}

\begin{abstract}
The demonstration of salt dissolution incorporated within powder-bed fusion fabrication processes has allowed the creation of complex porous structures without the need for sophisticated design algorithms. This serves to simplify the process, for porous structure creation in powder-bed fabrication techniques, creating a new opportunity for the realisation of optimised structures. A new methodology is presented here in which modulation of the energy density while using a single feedstock material enables three-dimensional control of porosity, ranging from $20 \%$ to $49 \%$. Through structured experimentation, the material's response to varying the process parameters in selective laser melting is evaluated and nested structures of distinct densities and morphologies are created. Correlation of the process parameters with modulus and ultimate compressive stress are established. A simple-assembly algorithm was used to generate complex parts consisting of locally assigned porosities having characteristic properties.
\end{abstract}

Keywords: Additive Manufacturing; cellular structures; selective laser melting, controlledporosity, mechanical performance, porous, graded materials

\section{Introduction}

Porogen or space holders are utilised in the powder metallurgy field where controlled porosity can be achieved through the thermal decomposition or dissolution of these space holders.Various materials have been processed in this manner, as reviewed extensively by Arifvianto et al. [1]. These include, but are not limited to, titanium alloys [2, 3], aluminium alloys [4], and stainless steels [5]. Commercial exploitation of these materials has been reported in biomedical [6], aerospace thermal, aerospace structural, acoustic, and filtration applications.

\footnotetext{
*Corresponding author: nesma.aboulkhair@nottingham.ac.uk
} 
The manufacture of porous materials, also known as cellular or lattice structures, by additive manufacturing (AM) remains a significant research area [7-9]. Typically, such structures are defined by the geometry of a repeating cell and its volume fraction. Generation of such structures requires specialist software and high computational expense and expert knowledge. The computational burden increases even further in the case of geometrical or volume fraction grading across the design domain. Additional to the difficulties in generating such structures for manufacture, there is also no simple and computationally efficient method of predicting their performance in order to optimise the design. In order to overcome these problems, a porogen based method has been proposed, which enables the creation of tailorable porous structures without the need to explicitly generate the detailed geometry [10]. In that work, a simple method was demonstrated for processing a metal-slat mixed feedstock within selective laser melting (SLM) in which the volume fraction, or density, could be varied by controlling the ratio of salt to metal powder. In this paper, this work is extended to demonstrate that volume fraction of porous structures can be varied through process, rather than feedstock, control, making it a significantly more attractive proposition for the manufacture of functionally or structurally graded parts. As a result, the shortcomings of the traditional route to lattice manufacture are overcome and graded porous structures can be easily created using laser powder-bed fusion, benefitting from the high degrees of freedom offered by the technology.

\section{Materials and Methods}

Plasma-atomized, spherical Ti-6Al-4V powder (Grade 5; LPW Technology Ltd) with a mean particle size of $\sim 35 \mu \mathrm{m}$ and sodium chloride ( $\mathrm{NaCl}, 98 \%$ pure-Peacock Salt) powder with a mean size, after sieving, of $\sim 150 \mu \mathrm{m}$ were used as the lattice material and space holder, respectively. Mixtures of Ti-6Al-4V and $\mathrm{NaCl}$ (40:60, by mass) were prepared by manual stirring followed by gyroscopic mixing for 30 minutes. A Realizer SLM-50 ${ }^{\circledR}$ selective laser melting machine, equipped with a $100 \mathrm{~W}$ Yttrium fibre laser, was utilised to fabricate samples with varying porosity by control of the processing parameters. The process chamber was flushed with Ar to generate a controlled atmosphere with less than $0.2 \%$ oxygen. The selectively laser melted samples were immersed in water at room temperature to leach out the $\mathrm{NaCl}$. X-ray Computed Tomography (XCT) measurements were performed using an Xradia 500 Versa X-ray microscope (XRM) to investigate, quantitatively and qualitatively, the relationship between porosity and process parameters. Further detailed description of the materials and methods used to manufacture the samples were reported previously [10]. Cuboidal samples $5 \times 5 \times 9 \mathrm{~mm}^{3}$ were fabricated using variable porosity along the height at 3 $\mathrm{mm}$ increments, as shown in Figure 1 ( $\mathrm{a}$ and $\mathrm{b}$ ). These samples were tested using an Instron 5969 universal testing machine at room temperature under compressive loading with a strain rate of $10^{-3} \mathrm{~s}^{-1}$.

\section{Results and Discussion}

Through varying the process parameters, marked changes in relative density were observed, as can be seen in Figure 1 (a-e). This was achieved whilst maintaining the same $\mathrm{NaCl}$ content in the powder, i.e. using the same feedstock material for all the samples whilst varying the process parameters, in contrast to [10] where the feedstock material was varied. The porosity content in a sample was found to be inversely related to the energy density deployed during processing 
where, as the energy density values decreased from $69.4-27.7 \mathrm{~J} / \mathrm{mm}^{3}$, porosity increased from $20 \%$ to $49 \%$. The reason for this is that the relative properties of the salt and metal powder means that a certain proportion of the salt is vaporised when melting the metal powder. A higher energy means a higher proportion of salt is vaporised which means it isn't available as a space holder the manufactured sample, and hence porosity will be less when the salt is removed.

In order to demonstrate the capability of producing a structurally graded part by altering the process parameters without changing the powder feedstock, $9 \mathrm{~mm}$ cubic samples were fabricated, assigning three sets of process parameters to distinct zones along the build direction (z-axis). The first, second, and third sections (3 mm thick each) were processed using R1 (69.4 $\left.\mathrm{J} / \mathrm{mm}^{3}\right), \mathrm{R} 2\left(55.5 \mathrm{~J} / \mathrm{mm}^{3}\right)$, and R3 $\left(27.7 \mathrm{~J} / \mathrm{mm}^{3}\right)$ energy densities, respectively, by changing the laser power and scan speed, producing respective porosities of $20 \%, 31 \%$, and $45 \%$. The density grading in the build direction is evident in the XCT 3D reconstruction (Figure 1 (a)). This experiment clearly shows that a controlled variation in the pore fraction can be achieved solely by changing the process parameters for a given feedstock material. This is consistent with the previously mentioned dependency of porosity on the energy density. There is a clear step change in the structure from one set of processing parameters to another, with a distinct transition between regions (Figure 1(b)), suggesting structural continuity during the change from one set of process parameters to another, which is essential for the parts to exhibit structural integrity. It was also seen that connectivity between the pores allowed efficient salt dissolution from the structure. As can be seen in Figure (1(f)), only a thin layer (below $10 \mu \mathrm{m}$ ) of residual $\mathrm{NaCl}$ remained on the internal periphery of the pore; this was confirmed using Energy Dispersive Spectroscopy (EDS). 

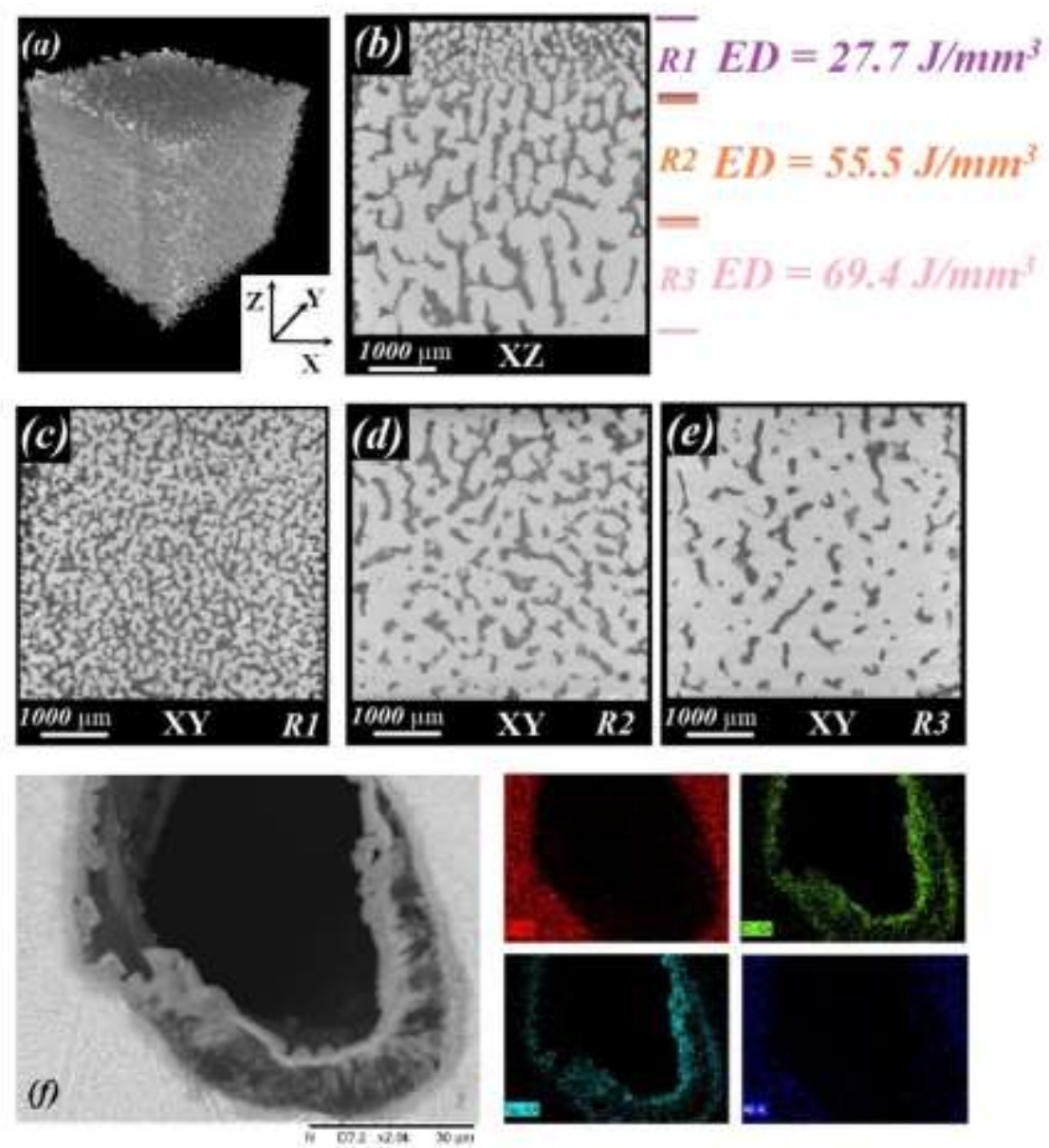

Figure 1: (a) A 3D reconstruction of an SLM part fabricated with graded porosity through controlling the process parameters developed from $\mu C T$ scans. (b) Cross-sectional view (XZ-plane) from a representative sample with graduated density achieved through layer-wise modulation of the process parameters revealing the three distinct regions $R 1, R 2$, and $R 3$ and (c-e) present XY-plane cross-sections from these regions. (f) EDS maps at a pore showing a thin layer of $\mathrm{NaCl}$ on the pore boundary. Porosity analysis is presented in $(g-h)$.

Another level of versatility that has not been demonstrated within powder-bed fusion is grading within an individual layer. Given the added advantage to this approach of not needing to change the powder feedstock, density variations within a layer (X-Y plane) through process parameter control was demonstrated by fabricating a simple nested structure. The component design was made up of a cylinder encased within a cuboid (Figure 2). The concept of nested structures here entails having discrete regions with different shapes in a build volume. In addition to this, we also demonstrate success in manufacturing a complex nested structure with an overhanging feature. In this case, the process parameters were modulated across the three axes X, Y, and Z (Figure 3). The letter "A" was fabricated with a high pore content ( $48.7 \%$ ) encapsulated in a denser casing (30.4\% porosity) with a gap within the letter " $\mathrm{A}$ " created by no laser scanning in the designated region. 

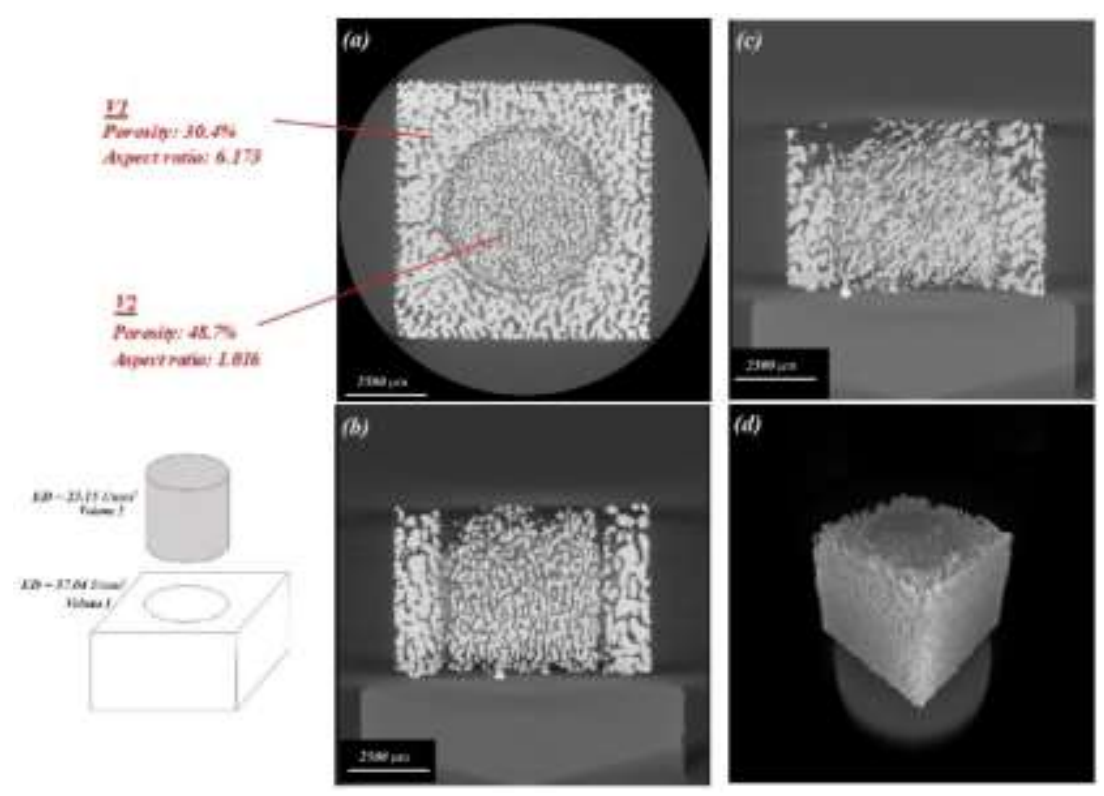

Figure 2: Simple assembly of structures and the assignment of localised process parameters allows the fabrication of parts with location-specific density using Ti-6Al-4V mixed with $60 \% \mathrm{NaCl}$. (a-c) show the three orthogonal views revealing the slices at the centre-line of the sample scanned by XCT and (d) shows the 3D reconstruction of the part.

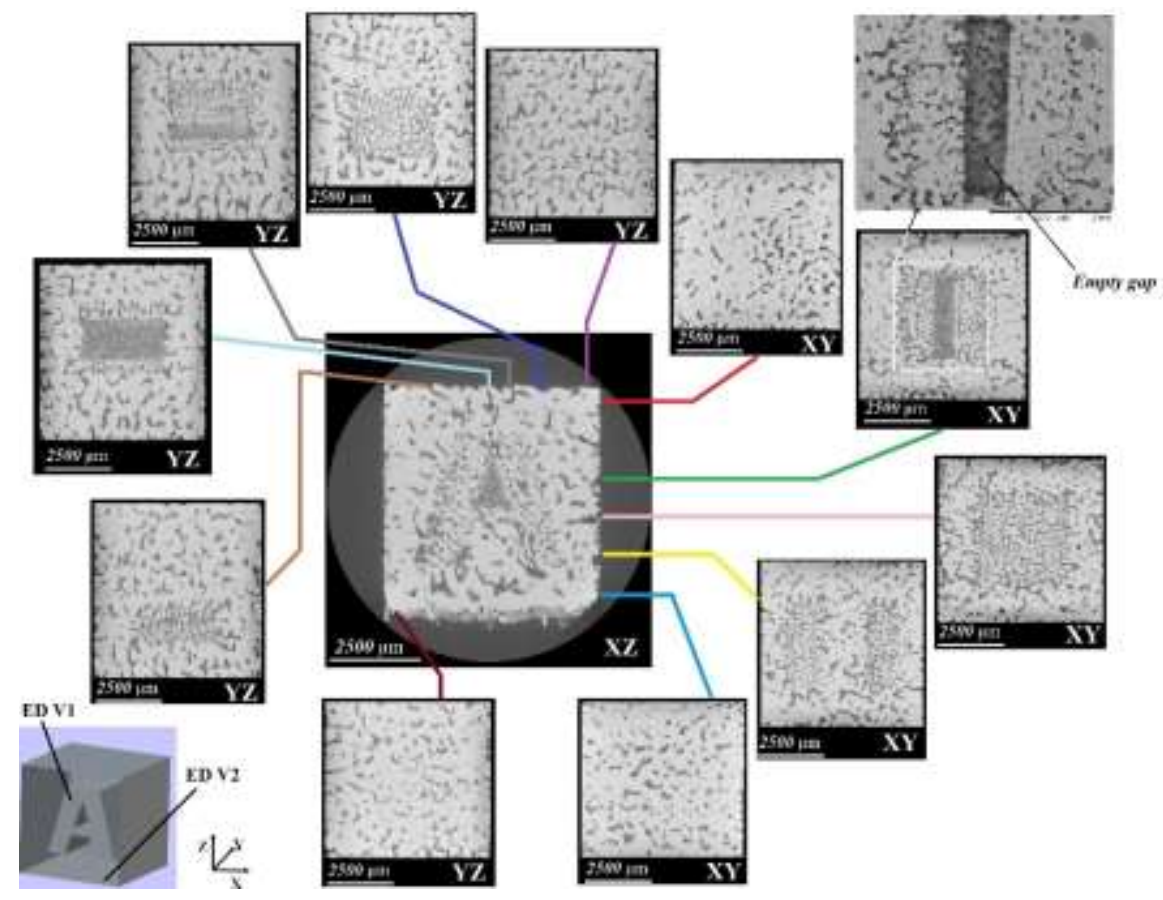

Figure 3: Here a letter ' $A$ ' is fully encased in a matrix of dissimilar porosity demonstrating the effect of distinct process parameters on resulting porosity. $\mu \mathrm{CT}$ scans across numerous regions and planes are presented; with an SEM image showing the fine features and the gap that was originally filled with loose non-molten powder for not being irradiated with the laser beam, the gap has become empty after cross-sectioning the sample.

The compressive behaviour of samples fabricated with uniform density has already been published in [10]. The uniform density samples failed in a brittle fashion through the formation of a diagonal shear band $45^{\circ}$ to the loading direction. As the porosity content increases, the mechanical strength deteriorates. Although this was observed for individual samples with consistent porosity in each, it can be related to the samples with graded density. Figure 4 shows a representative stress-strain curve for a sample having the same geometry and porosity 
distribution presented in Figure 1. It can be seen that the sample failed region-by-region in a sequential manner, identified in the figure as primary, secondary, and tertiary zones. The primary failure corresponds to the failure of the region in the sample with the highest porosity content, this being the weakest part of the specimen and the region fabricated using the lowest energy density, failing at approximately $325 \mathrm{MPa}$. The tertiary zone corresponds to failure in the strongest part of the specimen, fracturing at nearly $470 \mathrm{MPa}$, this being the region with the least porosity content, which was processed using the highest energy density. The secondary zone therefore, is the one with the intermediate properties and porosity, collapsing at $400 \mathrm{MPa}$. Between the primary and secondary phases, there is a readjusting or re-distribution of debris and bending of exposed struts. The ultimate compressive strength of the graded samples was comparable to that of the uniform density samples ( 450 MPa) reported previously for samples produced using the same feedstock [10]. The compressive strain, however, nearly tripled by graduating the porosity. Graduating the porosity content through process parameters modulation allows control of failure mode by design. The advantage of being able to control the material's performance and functionality within the manufacturing process grants the designers with an opportunity by which they can possibly engineer an object's response to an impact event.

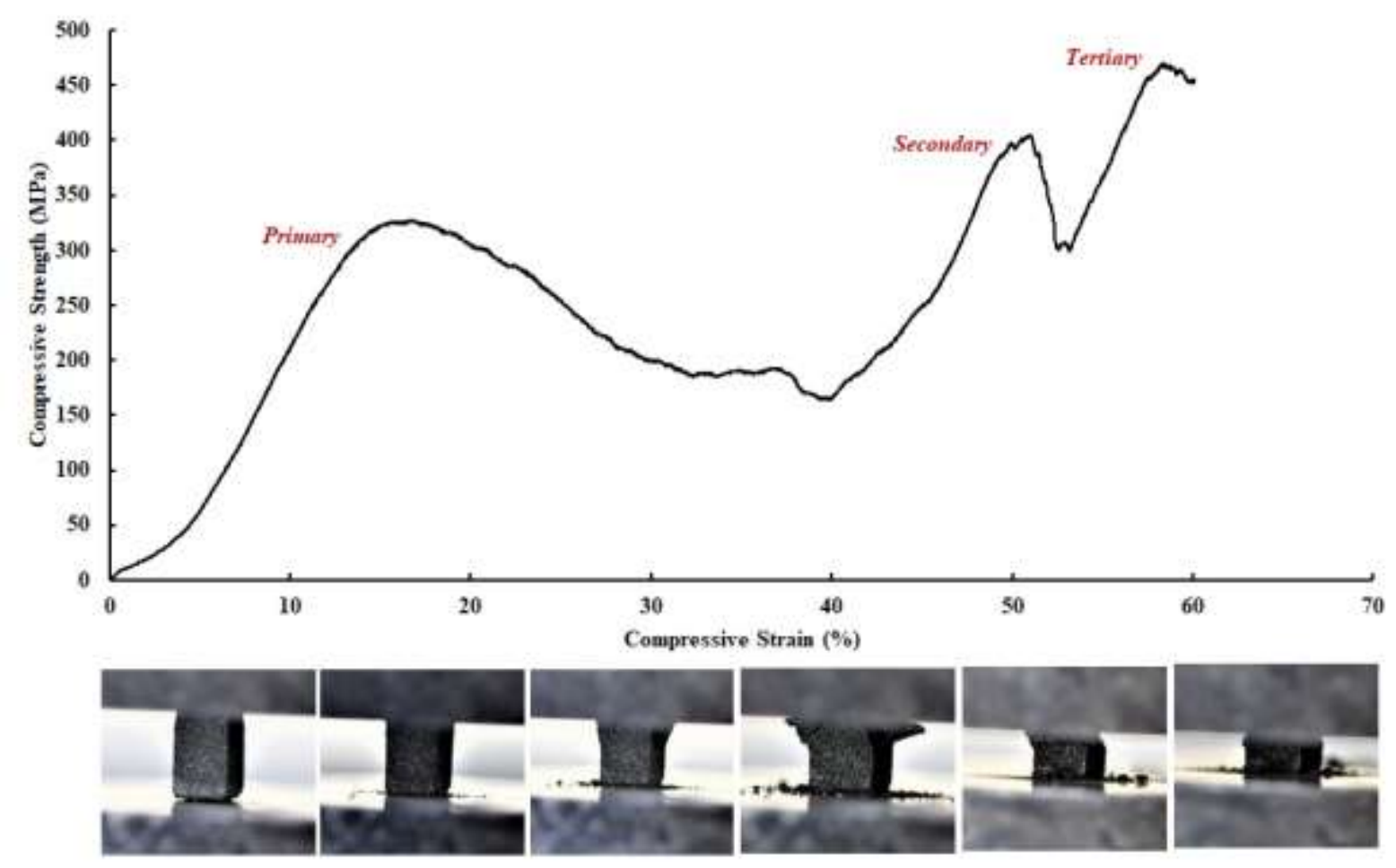

Figure 4: Representative stress-strain curve showing the mechanical performance of a graded structure under compressive loading.

\section{Summary and Conclusions}

Although in the literature varying the volume fraction of $\mathrm{NaCl}$ in the powder feedstock has proven successful in producing various levels of controlled porosity [10], this approach is problematic for the production of structurally-graded parts, as current class powder-bed fusion 
systems can only accommodate one feedstock material. It can be argued that density grading along the build direction can be achieved by changing feedstock powder in a layer-by-layer fashion. However, this will require significant manual intervention besides imposing severe difficulties in powder reuse.

This study presented a novel approach for the first time to exploit the correlation between process parameters and the amount of porosity developed in a selectively laser melted part. The approach enables not only density grading in the build direction (Z) without affecting the powder recyclability but also within individual layers (within the X-Y plane). A gradient of structural properties has been created by only adjusting laser energy density. Further in-depth studies are currently underway to investigate the correlation between altering the process parameters and the meso and microstructures produced in the samples fabricated.

Building upon previous work of the authors for the use of porogens within SLM, a method by which porosity can be controlled by varying only the energy density has been defined here. Through the correlation between the energy density and the resultant porosity, a set of process parameters can be defined for a given volume in a part to alter its porosity content for a given feedstock with a fixed salt (space holder) content. This has been demonstrated in this study through structurally grading the porosity content along the build direction (Z-axis) and for the first time using a powder-bed fusion process, within individual layers (X-Y plane) and hence concurrently in all three axes. The demonstrators reported in this article belonged to the macro scale as a proof of concept, where it is thought that there is strong potential for this to be translated to more complex structures with finer details and variations down to the single layer level, where there is potential for an almost continuous structural grading.

\section{References}

[1] Arifvianto B, Zhou J. Fabrication of Metallic Biomedical Scaffolds with the Space Holder Method: A Review. Materials (Basel, Switzerland). 2014;7:3588-622.

[2] Laptev A, Bram M. Manufacturing hollow titanium parts by powder metallurgy route and space holder technique. Materials Letters. 2015;160:101-3.

[3] Esen Z, Bor Ş. Processing of titanium foams using magnesium spacer particles. Scripta Materialia. 2007;56:341-4.

[4] Jiang B, Zhao NQ, Shi CS, Li JJ. Processing of open cell aluminum foams with tailored porous morphology. Scripta Materialia. 2005;53:781-5.

[5] Mondal DP, Jain H, Das S, Jha AK. Stainless steel foams made through powder metallurgy route using NH4HCO3 as space holder. Materials \& Design. 2015;88:430-7.

[6] Chen Y, Kent D, Bermingham M, Dehghan-Manshadi A, Wang G, Wen C, et al. Manufacturing of graded titanium scaffolds using a novel space holder technique. Bioactive Materials. 2017;2:248-52.

[7] Maskery I, Aboulkhair NT, Aremu AO, Tuck C], Ashcroft IA. Compressive failure modes and energy absorption in additively manufactured double gyroid lattices. Additive Manufacturing. 2017;16:24-9.

[8] Maskery I, Aremu AO, Simonelli M, Tuck C, Wildman RD, Ashcroft IA, et al. Mechanical Properties of Ti-6Al-4V Selectively Laser Melted Parts with Body-CentredCubic Lattices of Varying cell size. Exp Mech. 2015:1-12.

[9] Maskery I, Aboulkhair NT, Aremu AO, Tuck C], Ashcroft IA, Wildman RD, et al. A mechanical property evaluation of graded density Al-Si10-Mg lattice structures manufactured by selective laser melting. Materials Science and Engineering: A. $2016 ; 670: 264-74$. 
[10] Zhao G, Ashcroft IA, Hague RJM, Kennedy AR, Clare AT. Salt-metal feedstocks for the creation of stochastic cellular structures with controlled relative density by powder bed fabrication. Materials \& Design. 2018;149:63-72. 